\title{
Reducing Congestion in Real-Time Multi-party-tracking Sensor Network Applications
}

\author{
Wonwoo Jung, Sujeong Shin, Sukwon Choi, and Hojung Cha \\ Dept. of Computer Science, Yonsei University, \\ 134 Shinchon-dong, Seodaemun-gu, Seoul 120-749, Korea \\ \{wwjung, acid, sukwon, hjcha\}@cs.yonsei.ac.kr
}

\begin{abstract}
This paper presents a framework for a congestion reduction mechanism in multiple objects tracking applications. The multiple objects tracking application has a real-time characteristic, through which all packets should be delivered in as timely fashion as possible. The proposed framework consists of sink nodes, backbone nodes, mobile nodes and agent nodes. The location information of the mobile node is delivered to the sink node using both localization and routing techniques, which consider the network congestion. The proposed congestion reduction technique avoids network congestion by decreasing the number of transmitting packets based on the idea that network congestion occurs when the total data in transit is greater than the total channel capacity. The decrement is achieved by both merging and attachment operations, which consolidate several small packets into one large packet in intermediate backbone nodes. The mechanism also considers a time-constrained delivery to assure the user's service request. The simulation is conducted using a TOSSIM simulator, and the result shows that the proposed mechanism improves performance in terms of the successful delivery ratio and the delay time.
\end{abstract}

\section{Introduction}

Wireless sensor network applications are largely classified into non-real-time applications and real-time applications. The non-real-time application transmits information gathered by sensor nodes in the sensor field to sink nodes without timing constraints by using multi-hop routing technologies. In this application, information is often overlapped, thus the amount of information is considered more important than the accuracy of information Habitat monitoring is a representative application for a non-realtime application. The real-time application has a timing constraint: that is, the accuracy as well as the delivery time of information is important. Tracking mobile objects and monitoring the facility for disaster avoidance are good examples of real-time applications. Especially, tracking applications are considered the one of the major applications in sensor networks. In tracking applications, sensor nodes in the sensor fields track mobile objects, and transmit their location information to sink nodes. However, many of current approaches to track moving objects assume one or a few moving objects in the sensor field. As the sensor network becomes widely deployed, tracking applications should consider multiple objects for the practical reason. The application that tracks multiple mobile objects is called a Multi-Party Tracking application (MPT) in this paper. 


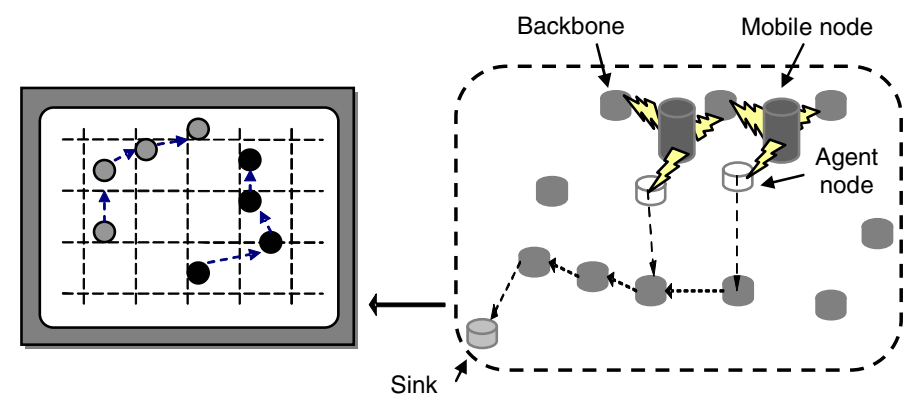

Fig. 1. Proposed framework for MPT applications

In MPT, as the number of moving object increases, the amount of information also increases, and this results in network congestion. A hop-by-hop flow control mechanism such as CODA (Congestion Detection and Avoidance) [1] and fairness control mechanism [2] reduces the congestion occurrence by reducing the transmission rate locally [3]. However, the mechanism also induces transmission terminations or delay. A rate limiting mechanism such as ESRT (Event-to-Sink Reliable Transport) [4] adjusts the reporting rate in order to reduce network congestion based on the idea that network congestion occurs when the total data in transit is greater than the total channel capacity [5]. However, the mechanism assumes the overlap of information, and does not consider time-constrained delivery. Fusion [3] combines the hop-by-hop flow control mechanism and the rate limiting mechanism, and Kang et al [6] proposed the mechanism that distributes packets to neighbor nodes in order to reduce network congestion. This research for managing network congestion has focused on reducing congestion occurrence. However, they overlooked both the user's service requirement and the time-constrained packet delivery. Moreover, this research is not suitable for MPT applications due to their dynamic adjustment of the event generation rate, because the MPT application expects to receive information with a fixed rate within a certain threshold.

This paper proposes a framework and a congestion reduction mechanism, called M\&A (Merge and Attach), for the MPT application. The mechanism adjusts the packet generation rate, and reduces the number of network packets by consolidating several small packets into one large packet at intermediate backbone nodes. It also considers a time constrained delivery, thus merging and attachment operations occur selectively based on real-time constraints. The paper is organized as follows. Section 2 describes the proposed framework and the congestion reduction technique. Section 3 discusses the experiment's results, and Section 4 concludes the paper.

\section{Congestion Reduction Mechanism for Multi-party-tracking}

The MPT application monitors the current location of various numbers of mobile nodes in sensor networks and transmits them to the sink node. Figure 1 shows the proposed framework of the MPT system. Since the MPT application is a real-time application, every information for location must arrive at the sink node within a certain deadline. In other words, location information must be transmitted immediately and the delay time occurring in the relaying process should be minimized. Other char- 
acteristics of MPT are a fixed reporting rate and single datum per single event, which means no redundant location information.

The proposed system consists of four types of sensor nodes. Mobile nodes, which calculate their location periodically using a localization algorithm, are attached to moving objects. Backbone nodes are aware of their global positions and are statically placed. When they receive beacon messages from mobile nodes, they calculate the distance between backbone nodes and mobile nodes, and relay the location information of mobile nodes to the sink node. Agent nodes, which are chosen by mobile objects, are backbone nodes, and relay the location information of mobile nodes to the sink node. Finally, the sink node is connected to the main system, and has an unlimited power source. The distance between the mobile node and the backbone node is calculated by the method used in the Cricket System [7], and the agent node is selected by the method used in SPEED [8]. The working scenario of MPT is followed. When multiple mobile nodes move, they broadcast beacon messages via RF and ultra sound periodically. Then, backbone nodes deployed in the sensor field receive the beacon message and calculate the distance between the backbone node and the mobile node. The calculated distance is transmitted to the mobile node, and then the mobile node selects three backbone nodes among them and calculates its location using the distance between the mobile node and the selected three backbone nodes. The location information is transmitted to the sink node through the agent node that is chosen from among three backbone nodes previously selected using multi-hop routing techniques. During the data transmission, the congestion reduction mechanism is conducted in order to reduce network traffic. The proposed system operates in two phases: the localization phase and the transport phase.

\subsection{Localization Phase}

Sampling rate $\mathrm{R}_{\mathrm{s}}$, which indicates the amount of transmitted data between a mobile node and a backbone node per unit time, is controlled by the mobile node according to the velocity change. $R_{s}$ is equal to or less than the reporting rate $R_{r}$, which is the amount of received data from a user per unit time, and decided by the user. The user receives the location information of the mobile node regardless of whether the data has actually arrived at the sink node or not. At the beginning of the tracking process, the mobile node calculates its own location with the initial sampling rate $\mathrm{R}_{\text {init }}$. The velocity is a vector value consisting of speed $v$ and direction $d$; therefore the velocity change is determined by both the speed change $\Delta v$ and the direction change $\Delta d$. Figure 2 shows the reconfigure process of $R_{s}$ depending on the velocity change of the mobile node.

When $\Delta v$ is larger than the speed threshold $\gamma_{\Delta v}$ (Case 1), $\mathrm{R}_{\mathrm{s}}$ is set to $\mathrm{R}_{\text {init }}$. When $\Delta d$ is larger than the direction threshold $\gamma_{\Delta d}$ (Case 2), $R_{s}$ is set to $R_{\text {init. }}$ In other cases (Case 3), $R_{s}$ is set to the default minimum value $R_{m}$. When the mobile node calculates its location, it decides whether the location information should be transmitted to the sink node or not, because the sink node can predict its location when the speed of the mobile node is moderate. Equation 1 and Equation 2 show the condition of the decision. 


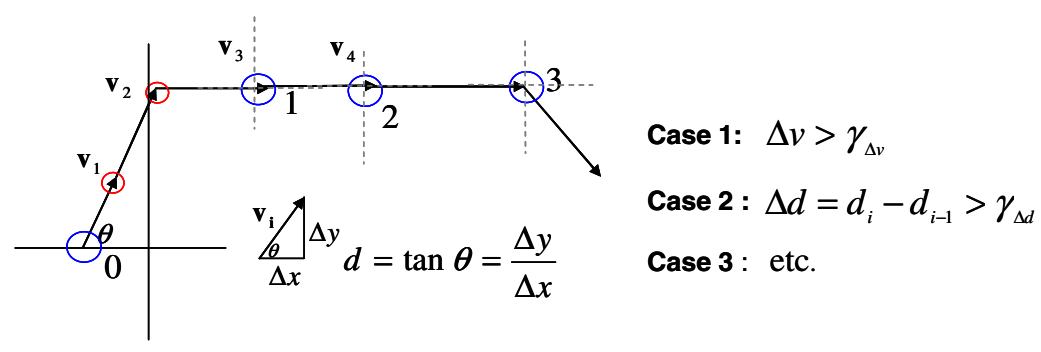

Fig. 2. Reconfiguration of the sampling rate

$$
\begin{gathered}
G\left(\mathbf{v}_{i-1}, \mathbf{v}_{i}\right)= \begin{cases}0 & \text { if } \mathbf{v}_{i}=\mathbf{v}_{i-1} \\
1 & \text { otherwise }\end{cases} \\
F\left(G\left(\mathbf{v}_{i-1}, \mathbf{v}_{i}\right), \Delta t\right)= \begin{cases}S E N D & \text { if } G\left(\mathbf{v}_{i-1}, \mathbf{v}_{i}\right)=1 \text { or } \Delta t \geq R_{m} \\
N O T \text { SEND } & \text { otherwise }\end{cases}
\end{gathered}
$$

When the mobile node decides to send the location information to the sink node, it selects one agent node, which has the biggest value of speed based on the SPEED [8] algorithm, from the backbone nodes (Equation 3). In Equation 3, Hop Delay indicates the elapsed time during the location information transmitted from the mobile node to the backbone node, and distance_mobile is the distance between the sink node and the mobile node. The distance between the sink node and the backbone node is denoted as distance_backbone. When the mobile node selects the agent node, it considers the distance as well as the communication ability of backbone nodes.

$$
\text { speed }=\frac{\text { distance_mobile }- \text { distance_backbone }}{\text { Hop Delay }}
$$

\subsection{Transport Phase}

When the agent node receives the location information of the mobile node, the second phase begins. Since MPT requires only 7 bytes for transmitting the location information of mobile nodes to the sink node in this phase, it is possible to consolidate several packets into one packet in order to reduce the network traffic because MICA2, operating on TinyOS, can transmit a packet with a size of 36bytes at the maximum [9]. Figure 3 shows the structure of the backbone node, which handles the merging and attachment operation (M\&A), and relays packets to the sink node. The merging operation consolidates several packets that are generated at a similar time, and the attachment operation suspends and attaches packets considering their delay time. The merging and attachment operation are applied separately based on two values: the elapsed time $\Sigma \Delta \mathrm{t}$, which means the duration between the current time and the packet creation time, and the threshold $\tau$. If the elapsed time is smaller than the threshold, which means the data is valuable in terms of the real-time concept, the merging operation is applied. Otherwise, the attachment operation is applied in order to give more precise location information for mobile nodes to the user; however, the data is out-of-date. 


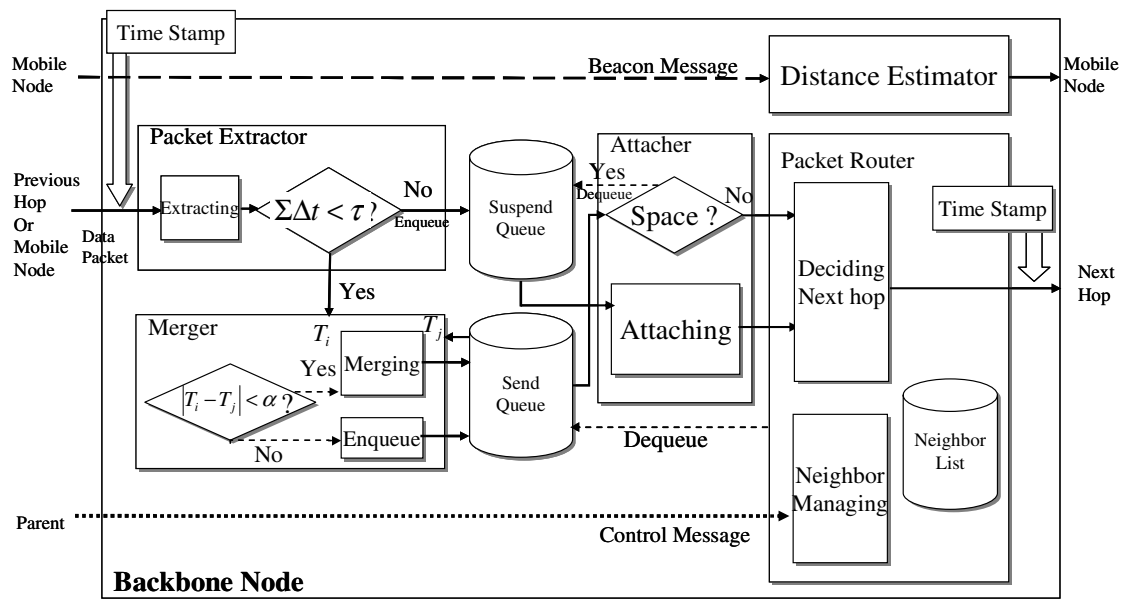

Fig. 3. Structure of the backbone node

The Packet Extractor compares $\Sigma \Delta \mathrm{t}$ and $\tau$, and determines which operation should be applied. If the merging operation is selected, the elapsed time of packets, which are stored at the send queue, are compared with one another. If the difference between them is within the range $\alpha$, the Packet Extractor examines the size of the packets and merges them if it is possible. When the difference exceeds $\alpha$, the merging operation is not applied. If the attachment operation is selected, the packet currently received does not have to be transmitted as soon as possible, because it cannot be delivered to the sink node on time; therefore it is stored at the suspend queue. These suspended packets are attached only if they can be attached to the packet that is going to transmit to the next node. Although attached packets cannot be delivered within a time constraint, they can give the user a hint about the moving track of the mobile node in the past.

\section{Evaluation}

Simulations were conducted with a TOSSIM [10] simulator of TinyOS, which is designed for MICA hardware. It simulates CC1000 [11] used at MICA2 selectively, and all simulations in the paper were conducted using $\mathrm{CC} 1000$ for a radio module. Currently, TOSSIM based on B-MAC [12] cannot use ACK messages. Kang et al. [13] showed that an ACK-based retransmission policy is required to fill a transmission buffer. Therefore, we modified the simulator to use ACK messages selectively. Table 1 shows simulation parameters and values used in the simulation.

With this setup, each backbone node has 4 direct-communicable neighbor nodes at its top, bottom, left, and right directions, because $\mathrm{CC} 1000$ has about a $15 \mathrm{~m}$ radio range. To emulate mobile nodes, some backbone nodes produce traffic. We adopted a simple routing policy, which selects the closest neighbor node to the sink node randomly. Based on the preliminary experiment, the maximum allowed time $\tau$, which is used to examine whether the real-time constraints of incoming packet at the sink node is satisfied, is set to $1000 \mathrm{~ms}$, which is the same as the reporting period. Because $\tau$, 
which is larger than the reporting period, decreases the delivery success ratio due to the increase of packet losses caused by send queue overflows. The merging boundary time $\alpha$, which is used to examine whether a new packet should be merged with other packets in the send queue or not, is set to $300 \mathrm{~ms}$, because small $\alpha$ decreases the delivery success ratio.

Table 1. Simulation parameters and values

\begin{tabular}{ll}
\hline Parameter & Value \\
\hline Distance between backbone nodes & $12($ Meter $)$ \\
Field size & $108 \times 108\left(\mathrm{~m}^{2}\right)$ \\
Number of backbone nodes & $10 \times 10$ \\
Number of simulations in each & 5 \\
Simulation time & 5 (Minutes) \\
Sink location & Center / Corner \\
Number of mobile nodes & 30 \\
Arrangement of mobile nodes & Random / Dense deployed \\
Size of send queue & $8 \times 36$ (Bytes) \\
Size of suspend queue & $32 \times 7$ (Bytes) \\
\hline
\end{tabular}

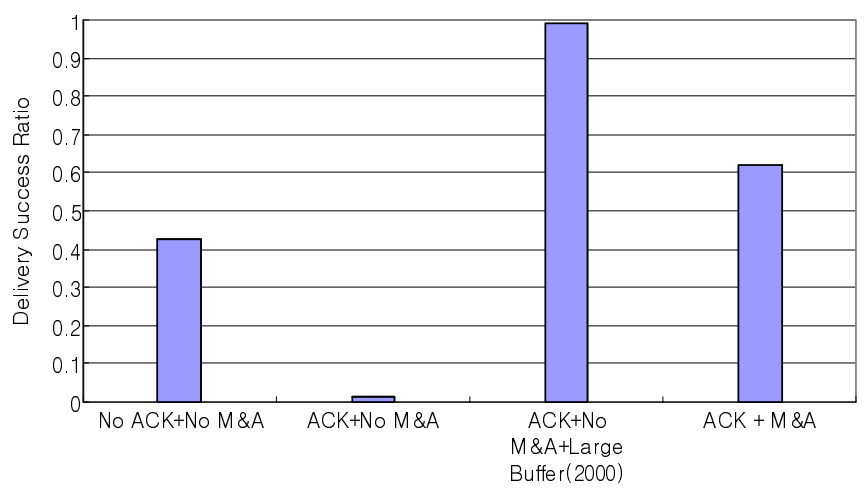

Fig. 4. Delivery success ratio in various settings

Figure 4 represents the delivery success ratio with 4 different cases according to the combination of two policies: ACK-based retransmission policy and M\&A policy. When the retransmission policy is not applied, the delivery success ratio is about $42.5 \%$, regardless of whether it is applying the M\&A policy or not. Since the M\&A policy utilizes packets in the transmission buffer, the retransmission policy should be applied to adopt the M\&A policy successfully. When the retransmission policy is applied without the M\&A policy, the delivery success ratio is about $1.5 \%$, which is the worst case of all. The worst delivery success ratio is due to the heavy traffic caused by retransmissions, which causes the transmission buffer to overflow. When the size of the transmission buffer is increased from $8 \times 36$ bytes to $2000 \times 36$ bytes, the 
delivery success ratio is about $100 \%$, and it is the best case of all. Although the large buffer improves the delivery success ratio, the elapsed time of almost every packet in this case is more than $35,000 \mathrm{~ms}$. Therefore, it is not applicable to MPT applications, which have a real-time characteristic. When the retransmission and M\&A policy are applied together, it achieves about a $62.0 \%$ delivery success ratio. Consequently, adopting both the ACK-based retransmission policy and M\&A policy achieve the best performance with the consideration of the real-time constraint.

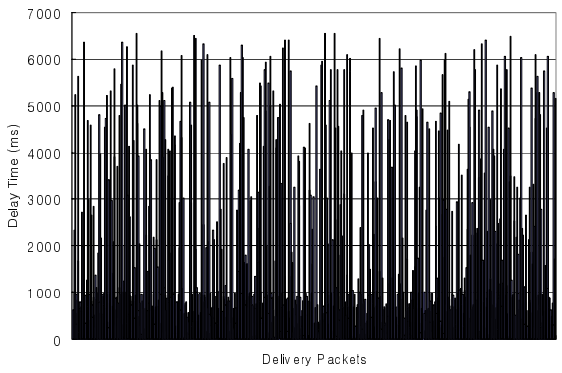

(a) M\&A with retransmission policy

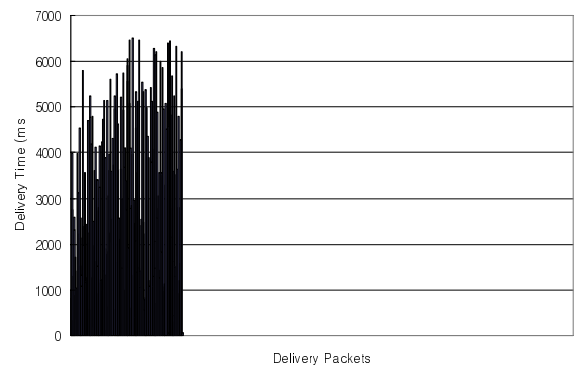

(b) Retransmission policy only

Fig. 5. Delay time distribution

Figure 5 shows the delay times of the first 1000 packets. Figure 5(a) represents the delay time when both the retransmission and M\&A policy are applied. In this case, about $60 \%$ of packets arrived at the sink node within the maximum allowed time $\tau, 1$ second. When the retransmission policy is applied without the M\&A policy, the delivery success ratio of this case is very low, and the delay time of arrived packets is higher than the other cases, as shown in Figure 5(b). When neither the retransmission nor the M\&A policy is applied, all packets successfully delivered to the sink node arrived within the allowed time. However, in this case, the delivery success ratio is about $40 \%$ lower than Figure 5(a). Therefore, applying both the M\&A policy and the retransmission policy achieve the best performance when considering both the delay time and the delivery success ratio.

Figure 6 shows the number of merging and attachment operations in the backbone nodes, when both the retransmission and M\&A policy are applied. The Z-axis shows the ratio of both merging and attachment operations. The position of each bar in Figure 6 is the same as the position of each node in the simulation. When nodes are located near to the event source, the merging operation occurs more frequently. In addition, the number of merging operations decreases, as nodes are located near to the sink node. On the contrary, the attachment operation occurs more frequently when nodes are located near to the sink node or if they are located far from the event source as Figure 6(b) shows. This is because the packet that has a long elapsed time has a high probability to attach. The high attachment operation ratio near the sink node reduces the traffic and the number of packets in the send queue. Therefore, the ratio of merging operating in this case decreases, because the attachment operation ratio is higher than the merging operation ratio near the sink node. 


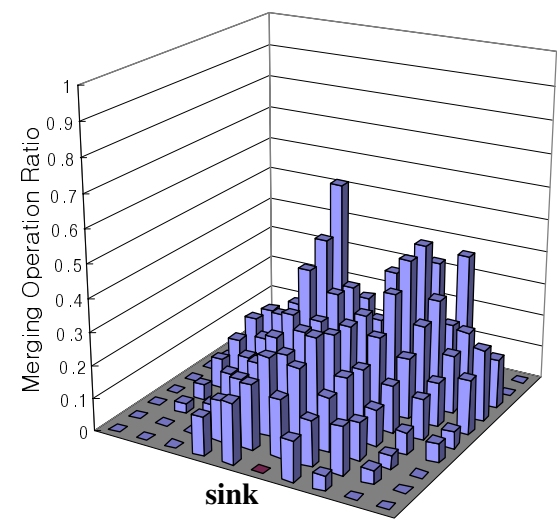

(a) Merge

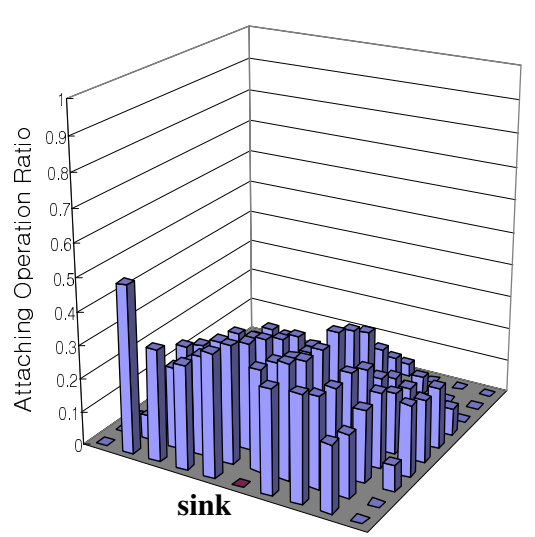

(b) Attach

Fig. 6. Merging and attachment operation ratio

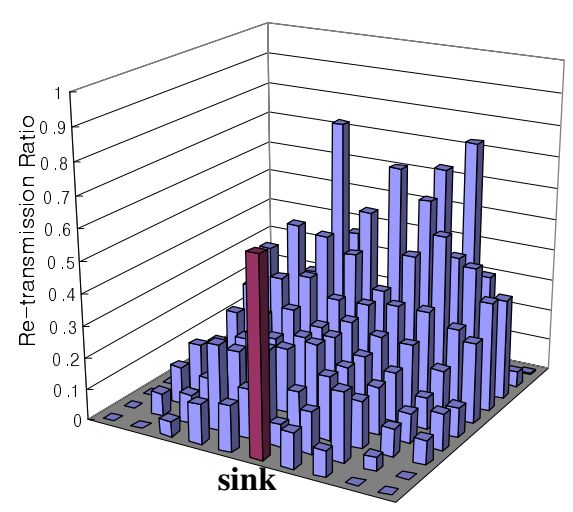

(a) Retransmission ratio with M\&A policy

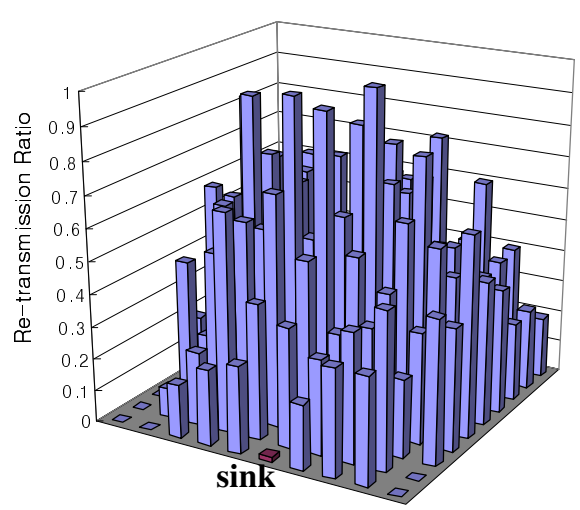

(b) Retransmission ratio without M\&A policy

Fig. 7. Retransmission ratio whether applying M\&A policy

Figure 7 shows the retransmission ratio whether applying M\&A policy or not. The $\mathrm{Z}$-axis in Figure 7 shows the retransmission ratio for one transmission at a node. In addition, the bar of the sink node represents the transmission success ratio. When the M\&A policy is not applied, as shown in Figure 7(b), the retransmission ratio increases when nodes are located near to the event source; it decreases as nodes are located nearer to the sink node. This is because the packet loss caused by the congestion reduces the network traffic, as nodes are located near to the sink node. When the M\&A policy is applied, as shown in Figure 7(a), the retransmission ratio decreases continuously, as nodes are located near to the sink node. It proves that the merging and attachment operations reduce network congestion occurrences.

Figure 8 shows the total number of transmitted packets and the amount of transmitted data. The total number of transmitted packets decreases as packets are transmitted 
toward the sink node. On the other hand, the total amount of transmitted data increases as packets are transmitted toward the sink node. When the M\&A policy is applied, the transmitted packets are reduced by $26 \%$ of the total amount of data. It proves that the merging and attachment operation reduces the total number of packets even though the amount of data is increased.

As a result, the proposed M\&A policy shows the improvement of the delivery success ratio, when it is applied with the retransmission policy. Although performance in terms of the delay time may be worse than when it is applied without the retransmission policy, the degradation is trivial. Moreover, from the maximum allowed time of view, it achieves a good performance.

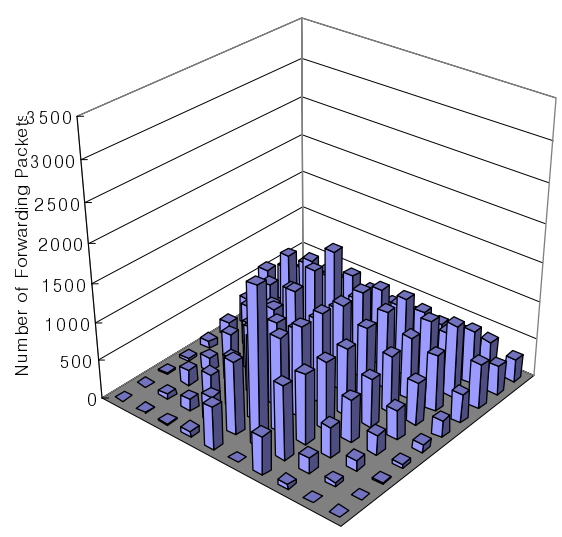

(a) Forwarding packets

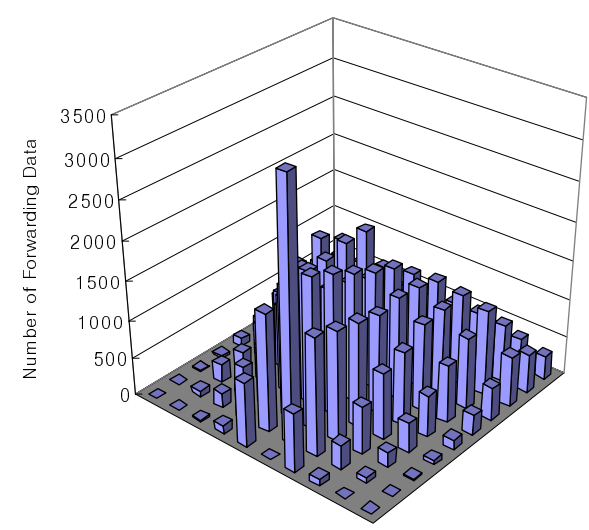

(b) Forwarding data

Fig. 8. Number of forwarding packets and amount of forwarding data

\section{Conclusions}

There are a number of approaches to solving the congestion problem in sensor networks; however, they cannot be applied to MPT application because of its real-time constraints. M\&A uses a merging or attachment operation in order to decrease the number of packets in networks. Because the M\&A mechanism does not suppress the data generation rate and does not postpone the forward of packets, it is suitable for real-time applications. The simulation result shows that using the M\&A mechanism improves the delivery success ratio more than any other cases. The delay time is also examined in order to evaluate real-time constraints. When using the M\&A mechanism with the retransmission mechanism the delay time is a little longer than without using the retransmission mechanism. However, it does not mean that the M\&A mechanism with the retransmission mechanism performs badly in terms of real-time constraints, since packets that arrived at the sink node within $\tau$ are considered as packets that satisfy real-time constraints in MPT applications. From this point of view, we can conclude that the case with M\&A and the retransmission mechanism showed similar performance to the case without the retransmission mechanism. 
We are implementing the proposed framework and M\&A mechanism to real hardware based on MSP430 MPU. Our final goal is to implement the MPT application on real hardware, including the congestion mechanism and localization algorithms for tracking itself.

\section{Acknowledgements}

This work was supported by the National Research Laboratory (NRL) program of the Korean Science and Engineering Foundation (2005-01352) and the ITRC Program (MMRC) of IITA, Korea.

\section{References}

1. C.-Y. Wan, S. B. Eisenman, and A. T. Campbell.: CODA: Congestion Detection and Avoidance in Sensor Networks. In Proc. of ACM SenSys 2003, Los Angeles, USA, (2003)

2. C.-T. Ee and R. Bajcsy.: Congestion Control and Fairness for Many-to-One Routing in Sensor Networks. In Proc. ACM SenSys 2004, Baltimore, MD, (2004)

3. B. Hull, K. Jamieson, and H. Balakrishnan.: Mitigating Congestion in Wireless Sensor Networks. In Proc. of ACM SenSys 2004, Baltimore, MD (2004)

4. Y. Sankarasubramaniam, O. B. Akan, and I. F. Akyildiz.: ESRT: Event-to-Sink Reliable Transport in Wireless Sensor Networks. In Proc. of MobiHoc 2003, Annapolis, Maryland (2003)

5. S. Tilak, N. B. Abu-Ghazaleh, and W. Heinzelman.: Infrastructure Tradeoffs for Sensor Networks. In Proc. of WSNA 2002, Atlanta (2002), 49-58

6. J. Kang, Y. Zhang, B. Nath, and S. Yu.: Adaptive Resource Control Scheme to Alleviate Congestion Control in Sensor Networks. In Proc. of the 1st Workshop on Broadcast Advanced Sensor Networks (BASENETS), San Jose, CA (2004)

7. N. B. Priyantha, A. Chakraborty, and H. Balakrishnan.: The Cricket Location-Support System, In Proc. of 6th ACM MOBICOM, Boston, MA (2000)

8. T. He, J. A. Stankovic, C. Lu, and T. F. Abdelzaher.: SPEED: A Stateless Protocol for Real-Time Communication in Sensor Networks. In Proc. of International Conference on Distributed Computing Systems (ICDCS 2003), Providence, RI (2003)

9. http://www.tinyos.net

10. P. Levis, N. Lee, M. Welsh, and D. Culler.: Tossim: Accurate and Scalable Simulation of Entire Tinyos Applications, In Proc. of ACM SenSys 2003, Los Angeles, USA (2003)

11. http://www.chipcon.com/files/CC1000_Data_Sheet_2_2.pdf

12. J. Polastre, J. Hill, and D. Culler.: Versatile Low Power Media Access for Wireless Sensor Networks, In Proc. ACM SenSys 2004, Baltimore, MD (2004)

13. J. Kang, Y. Zhang, and B. Nath.: Accurate and Energy-efficient Congestion Level Measurement in Ad Hoc Networks, In Proc. of the IEEE Wireless Communications and Networking Conference (WCNC), New Orleans, LA (2005) 